\title{
Immunotherapy: new options in breast cancer treatment
}

\author{
Michael Untch ${ }^{\dagger}$ Nina Ditsch and Kerstin Hermelink
}

\section{CONTENTS}

Active specific immunotherapy: Theratope ${ }^{\circledR}$

Monoclonal antibodies against HER-2: trastuzumab

Expert opinion

Five-year view

Key issues

References

Affiliations

\footnotetext{
${ }^{\dagger}$ Author for correspondence Department of Gynecology and Obstetrics,

Ludwig-Maximilians University, Klinikum Munich-Grosshadern, Marchioninistr 15, D-81377,

Munich, Germany

muntch@.med.uni-muenchen.de
}

KEYWORDS

active specific immunotherapy, HER-2, metastatic breast cancer, passive immunotherapy, Theratope, trastuzumab

Immunotherapy is a promising new approach in breast cancer treatment, complementing surgery, chemotherapy, radiation and antihormonal therapy. Primarily, two treatment options are available which are supported by a rapidly growing body of clinical evidence. These are active specific immunotherapy with Theratope ${ }^{\circledR}$ and passive immunotherapy targeting the HER-2 receptor with trastuzumab (Herceptin ${ }^{\circledR}$ ). Trastuzumab has a proven efficacy as monotherapy as well as in combination with chemotherapeutic agents in HER-2-overexpressing metastatic breast cancer. Trastuzumab is generally well tolerated although cardiotoxicity has been observed, especially when in combination with doxorubicin (Adriamycin ${ }^{\circledR}$ ), where this can be a serious concern. Therefore, less cardiotoxic combinations with docetaxel $\left(\right.$ Taxotere $^{\circledR}$ ), vinorelbine $\left(\right.$ Navelbine ${ }^{\circledR}$ ) and epirubicin (Pharmorubicin ${ }^{\circledR}$ ) or cyclophosphamide (Endoxana ${ }^{\circledR}$ ) have been tested. The combination of trastuzumab with paclitaxel $\left(\operatorname{Taxol}^{\circledR}\right)$ is well approved. The use of trastuzumab in adjuvant and preoperative therapy is currently being examined in controlled trials. After a brief outline of immunotherapy with Theratope and trastuzumab, this article reviews recent and ongoing clinical studies conducted with trastuzumab.

Expert Rev. Anticancer Ther. 3(3), 403-408 (2003)

Active specific immunotherapy: Theratope ${ }^{\circledR}$ The cancer vaccine Theratope ${ }^{\circledR}$ (Biomira Inc., Alberta, Canada) stimulates an immune response against the tumor-associated antigen Sialyl-Tn (STn), a carbohydrate that associates with the mucin MUC1 overexpressed in breast cancer cells. STn is associated with more aggressive disease and poor prognosis. Theratope consists of a synthetic STn antigen emulating the tumor cell STn and a carrier molecule which presents the antigen to the immune effector cells, thus triggering antiSTn antibodies as well as mucin-specific T-cell responses [1].

A Phase II study has tested Theratope in metastatic breast cancer patients with STn expression [2]. A significant survival benefit could be demonstrated for patients treated with a combination of intravenous cyclophosphamide (Endoxana ${ }^{\circledR}$, Baxter, IL, USA) and Theratope. There was no correlation in the antibody response to the carrier molecule and survival. This led to the assumption that the observed survival benefit is based upon the specific antibody response to the synthetic mimic of the
STn carbohydrate incorporated in the Theratope vaccine. A review of clinical trials conducted with Theratope has been published [3].

\section{Monoclonal antibodies against}

HER-2: trastuzumab

The HER-2/neu oncoprotein

HER-2 is a transmembrane tyrosine kinase receptor belonging to the family of epidermal growth factor receptors (FIGURE 1). It is encoded by the HER-2/neu proto-oncogene located on chromosome 17q21 [4]. HER-2 is overexpressed in approximately $25-30 \%$ of all breast cancers [5]. Amplification of HER-2/neu gene and overexpression of the HER-2 protein stimulates cancer cell growth and is therefore of high prognostic relevance.

HER-2 overexpression correlates with a shorter relapse-free interval and overall survival time [6]. The unfavorable prognosis associated with HER-2 overexpression has been confirmed by numerous studies [7].

HER-2 overexpression is determined by immunohistochemistry. Currently, the most 


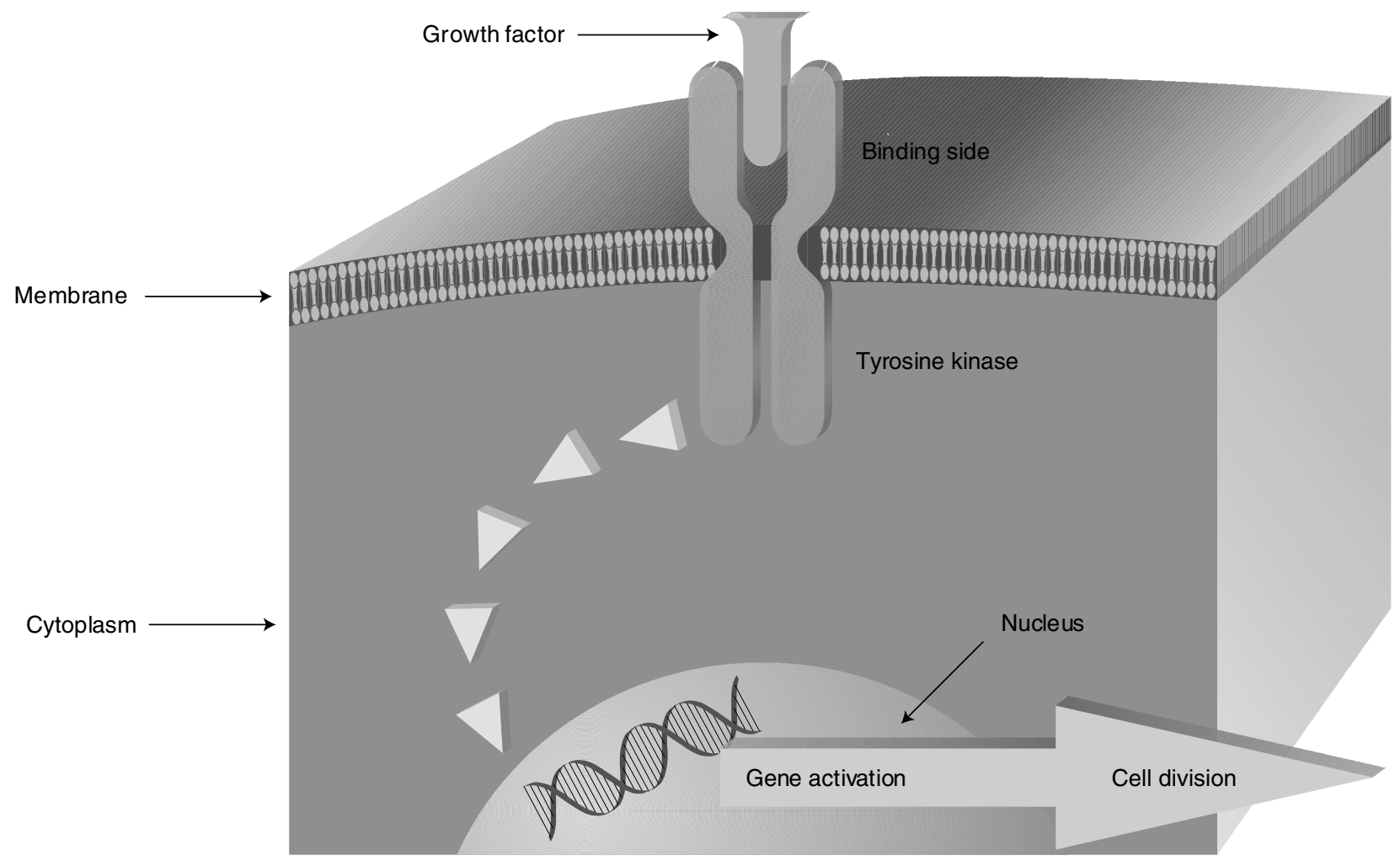

Figure 1. The HER-2 growth factor receptor.

commonly used test is the HercepTest ${ }^{\circledR}$ (Genentech, CA, USA), originally developed by Dako (Glostrup, Denmark) using a polyclonal antibody against HER-2/neu. A HER-2 score of 0 and $1+$ is classified as negative and $3+$ is clearly positive. In the case of a HER-2 score of $2+$ (marginally positive) a fluorescence in situ hybridization (FISH) analysis should follow for exact determination. Accurate determination of HER-2 status is an indispensable prerequisite to every trastuzumab (Herceptin ${ }^{\circledR}$, Genentech) treatment.

\section{Therapy of HER-2-overexpressing breast cancer with trastuzumab}

Trastuzumab is a monoclonal antibody $(\mathrm{mAb})$ that targets the extracellular domain of the HER-2 protein. The response to therapy correlates with HER-2 overexpression. Trastuzumab is indicated clearly in patients with immunohistochemical scores of $3+$ or more. In case of a 2+ score, FISH analysis should clarify amplification of the HER-2/neu gene [8-10].

In the USA, trastuzumab was approved for the treatment of metastatic breast cancer in 1998 and European approval followed in 2000. Phase II and III studies demonstrated efficacy and tolerability of trastuzumab in metastatic breast cancer patients with HER-2 overexpression (score $2+$ and $3+$ ). In these trials, trastuzumab has been used either as monotherapy or in combination with various chemotherapeutic agents. The treatment dose of trastuzumab is $2 \mathrm{mg} / \mathrm{kg} /$ week following a loading dose of $4 \mathrm{mg} / \mathrm{kg}$. A trastuzumab treatment regimen of 3-weekly $8 \mathrm{mg} / \mathrm{kg}$ loading dose followed by $6 \mathrm{mg} / \mathrm{kg}$ is also being tested.

\section{Trastuzumab monotherapy}

Cobleigh and colleagues treated 222 metastatic breast cancer patients with trastuzumab as second- or third-line therapy (68\% had previously received chemotherapy) [11]. The overall response rate was $15 \%$ (6\% with a score of $2+, 18 \%$ with a score of 3+) with a median time-to-progression of 9.1 months and a median survival of 13 months. There were eight complete responses (CRs) and 26 partial remissions (PRs). Of the patients, $40 \%$ suffered mild toxicity and cardiac side effects were observed in ten patients (4.7\%), of which nine were pretreated with doxorubicin (Adriamycin ${ }^{\circledR}$, Pharmacia, NJ, USA).

Vogel and coworkers treated 114 metastatic breast cancer patients with two different dosages of trastuzumab [12]. The first group received a loading dose of $8 \mathrm{mg} / \mathrm{kg}$ and a $4 \mathrm{mg} / \mathrm{kg}$ maintaining dose, while the second group received $4 \mathrm{mg} / \mathrm{kg}$ followed by $2 \mathrm{mg} / \mathrm{kg}$. Treatment was well tolerated in both treatment groups. Toxicity was more pronounced in the dose-intensified group but generally mild. The overall response rate was $26 \%$ without a significant difference between groups $(24 \%$ standard dose, $28 \%$ dose-intensified). Subgroup analysis demonstrated an elevated response rate in immunohistochemistry $(3+, 35 \%)$ and in FISH-positive patients (41\%). The median survival was 24.4 months. 
Table 1. Results of the H0650 and H0649 studies determining HER-2 overexpression by fluorescence in situ hybridization and immunohistochemistry.

\begin{tabular}{|c|c|c|c|}
\hline Trial parameters & $\mathrm{FISH}+$ & FISH- & $\mathrm{IHC}+$ \\
\hline \multicolumn{4}{|l|}{ H0650g } \\
\hline Response rate (\%) & 34 & 7 & 26 \\
\hline $\mathrm{CR}+\mathrm{PR}+\mathrm{SD}(\%)$ & 48 & 10 & 38 \\
\hline Overall survival (months) & 24.5 & 24.4 & 24.4 \\
\hline \multicolumn{4}{|l|}{ Ho649g } \\
\hline Response rate (\%) & 19 & 0 & 15 \\
\hline$C R+P R+S D(\%)$ & 33 & 6 & 28 \\
\hline Overall response (months) & 14.2 & 8.8 & 12.8 \\
\hline
\end{tabular}

Current data suggest that FISH analysis predicts the benefit from trastuzumab therapy more accurately and selection of patients should therefore be based upon this test [13]. Two studies with trastuzumab monotherapy assessed correlations of positive test results and therapy benefit. In the first study, $\mathrm{H} 0650 \mathrm{~g}$, patients were treated with trastuzumab as first nonhormonal therapy for metastatic disease, and in the second, $\mathrm{H} 0649 \mathrm{~g}$, patients who displayed disease progression after one or two previous chemotherapies were enroled. The correlation of response rate and a positive FISH test result was 34\% $(\mathrm{H} 0650 \mathrm{~g})$ and $19 \%(\mathrm{H} 0649 \mathrm{~g})$, compared with a correlation with immunohistochemically determined HER-2 overexpression of only 26 and $15 \%$, respectively (TABLE 1 ). If CR, PR and stable disease (SD) are considered together, the differences are similar.

\section{Trastuzumab in combination therapies}

First results of a treatment combining paclitaxel $\left(90 \mathrm{mg} / \mathrm{m}^{2} /\right.$ week $)$ and trastuzumab were published in 1999 and the response rate was $61 \%$ [14].

Slamon and colleagues compared the treatment effects of chemotherapy alone versus chemotherapy and trastuzumab combined in a sample of 469 patients with HER-2-overexpressing tumors and without previous treatment for metastatic breast cancer [15]. Patients were randomized to one of four treatment regimens:

- Trastuzumab plus an anthracycline and cyclophosphamide: doxorubicin $60 / 600 \mathrm{mg} / \mathrm{m}^{2}$ or epirubicin $75 / 600 \mathrm{mg} / \mathrm{m}^{2}$ for six doses every 3 weeks
- An anthracycline and cyclophosphamide alone

- Trastuzumab plus paclitaxel (Taxol ${ }^{\circledR}$, Bristol-Myers Squibb, NY, USA), $175 \mathrm{mg} / \mathrm{m}^{2}$ for six doses every 3 weeks

- Paclitaxel alone, $175 \mathrm{mg} / \mathrm{m}^{2}$

The trial demonstrated favorable effects of trastuzumab on response rate, median time-to-progression, median duration of response and survival. Overall responses rates to chemotherapy plus trastuzumab versus chemotherapy alone were 50 and $32 \%$, respectively, $(\mathrm{p}<0.001)$. The combination therapy with anthracycline chemotherapy plus trastuzumab demonstrated an increased response rate of 56 versus $42 \%$ for the same chemotherapy regimen without trastuzumab $(p=0.02)$. Response rates to trastuzumab plus paclitaxel versus paclitaxel alone were 41 and $17 \%$, respectively, $(p<0.001)$. Both trastuzumab groups demonstrated an improved time-to-progression compared with groups receiving chemotherapy alone. Time-to-progression was 7.8 months for anthracycline/cyclophosphamide plus trastuzumab versus 6.1 months for anthracycline/cyclophosphamide alone ( $\mathrm{p}$ $<0.001$ ) and 6.9 months for paclitaxel plus trastuzumab versus 3.0 months for paclitaxel alone $(\mathrm{p}<0.001)$.

The median survival time of all 469 patients was 9.1 months with trastuzumab versus 6.1 months without trastuzumab $(p<0.001)$. For the different treatment arms, anthracycline/cyclophosphamides plus trastuzumab was 9.1 months versus 6.7 months, $(\mathrm{p}=0.005)$ and for paclitaxel plus trastuzumab was 10.5 months versus 4.5 months $(p<0.01)$. Trastuzumab therefore improved the median survival time by 4.8 months compared with chemotherapy alone $(\mathrm{p}=0.046)$.

Table 2. Response rate, clinical benefit and time-to-disease progression in correlation with HER-2 serum antigen [21].

\begin{tabular}{lrrr}
\hline Parameters & HER-2/neu-positive & HER-2/neu-negative & Significance \\
\hline Objective response rate (\%) & 15 & 31 & $p=0.0001$ \\
Clinical benefit (\%) & 30 & 50 & $p=0.0001$ \\
Time-to-progression (months) & 4.2 & 9.2 & $p=0.0001$
\end{tabular}




\section{Box 1. Ongoing trastuzumab studies.}

- The National Surgical Adjuvant Breast Project trial B-31 uses a 3-weekly anthracycline/cyclophosphamide treatment, for four cycles, followed by four cycles of 3weekly paclitaxel plus trastuzumab versus the same chemotherapy regimen without trastuzumab in nodepositive, HER-2-positive breast cancer patients. Over a period of 5 years, 2700 patients will be enrolled. To date, more than 1200 patients have been accrued.

- The North Central Cancer Treatment Group trial N9831 is a randomized three-arm trial. Treatment with 3-weekly anthracycline/cyclophosphamide for four cycles in all three arms is followed by weekly paclitaxel for 12 cycles versus weekly paclitaxel for 12 cycles plus trastuzumab for 1 year versus weekly paclitaxel and trastuzumab for 12 cycles followed by 40 cycles of trastuzumab monotherapy. Patients have node-positive or high-risk node-negative breast cancer with overexpression of HER-2. To date, more than 1400 patients have been recruited.

- In the Breast Cancer International Research Group trial 006, patients with node-positive or high-risk node-negative, HER-2-overexpressing primary breast cancer are randomized to one of three treatment arms: four cycles of 3-weekly doxorubicin and cyclophosphamide, followed by either four cycles of 3-weekly docetaxel, four cycles of 3-weekly docetaxel plus trastuzumab for 1 year, or docetaxel plus carboplatin for six cycles, followed by trastuzumab for 1 year. The study has now accrued more than 1600 patients.

- The HERceptin Adjuvant (HERA) study is a multicenter study in which patients with verified HER-2 overexpression are randomly assigned to three different treatment groups: trastuzumab 1 year, 3-weekly after optimal primary therapy, trastuzumab 2 years, 3-weekly after optimal primary therapy or optimal primary therapy only (surgery, radiotherapy or endocrine therapy).

Symptomatic and asymptomatic cardiac dysfunctions were observed in 63 patients. Of these, $16 \%$ who had been treated with an anthracycline/cyclophosphamide and trastuzumab combination developed a New York Heart Association class III or IV dysfunction, 3\% with anthracycline/cyclophosphamide alone, $2 \%$ with paclitaxel and trastuzumab, and $1 \%$ with trastuzumab alone.

Two studies have researched combination therapy with trastuzumab. The first is with vinorelbine (Navelbine ${ }^{\circledR}$, GlaxoSmithKline, London, UK) and the second (M77003), is with epirubicin/cyclophosphamide as first-line therapy in anthracycline-naive metastatic breast cancer patients.

In the first study, $40 \mathrm{HER}$-2-positive patients with metastatic disease received $25 \mathrm{mg} / \mathrm{m}^{2}$ vinorelbine every week with a loading dose of $4 \mathrm{mg} / \mathrm{kg}$ trastuzumab [16]. Of the patients, $82 \%$ had previous adjuvant $(30 \%)$ or palliative $(25 \%)$ therapy, $20 \%$ had previously received anthracyclines, $15 \%$ taxanes and $38 \%$ anthracyclines and taxanes. The overall response rate was $75 \%$ and the combination therapy was well tolerated. The most common hematological side effect was neutropenia and three patients developed Grade 2 cardiotoxicity.

In the first part of Phase II of the second study, 26 HER-2/neu patients were treated with $60 / 600 \mathrm{mg} / \mathrm{m}^{2}$ epirubicin/cyclophosphamide for four doses, every 3 weeks, plus trastuzumab every week until week 103 , with an overall response of $71 \%$. In the second part, $25 \mathrm{Her}-2 /$ neu-positive patients were treated with an elevated dose of $90 / 600 \mathrm{mg} / \mathrm{m}^{2}$ epirubicin/cyclophosphamide and trastuzumab for 103 weeks. HER-2/neu-negative patients were treated as a control group (25 patients) with the same dose for four doses every 3 weeks without trastuzumab. In this study, cardiac function was monitored closely. A total of eight patients had a left ventricular ejection fraction (LVEF) decrease of more than $10 \%$, however, no patients demonstrated a decrease of more than $50 \%$. No patients developed a symptomatic change of the LVEF [16]. In the subsequent and ongoing Phase III study, $75 \mathrm{Her}-2 /$ neu positive patients are treated with $60 / 600$ or $90 / 600 \mathrm{mg} / \mathrm{m}^{2}$ epirubicin/cyclophosphamide every 3 weeks followed by trastuzumab until progression. Additional Her-2/neu neagative patients (75) will receive $90 / 600 \mathrm{mg} / \mathrm{m}^{2}$ epirubicin/cyclophosphamide. Results are not yet available.

The efficacy of trastuzumab with paclitaxel is currently being examined in the Cancer and Leukemia Group B (CALGB) 9840 study (weekly vs. 3-weekly trastuzumab plus paclitaxel).

\section{Irastuzumab \& antihormonal therapy}

According to the results of several studies, HER-2/neu overexpression is associated with resistance to antihormonal therapy [17]. The International Breast Cancer Study Group analyzed the data of 1506 patients and found a correlation of HER-2 overexpression and negative hormone receptor (HR) status.

Both Borg and coworkers, and Ravdin and colleagues reported a decreased effect of tamoxifen in HR-positive and HER-2-overexpressing patients [18,19].

Mass and colleagues presented similar clinical results of estrogen receptor (ER)-negative, HER-2-positive, as well as ER-positive, HER-2-positive patients treated with trastuzumab alone or in combination with chemotherapy [20].

Lipton and colleagues indicated that elevated HER-2 serum antigen correlates with decreased efficacy and shorter survival of breast cancer patients with metastatic disease treated with tamoxifen compared with letrozole $\left(\right.$ Femara $^{\circledR}$, Novartis, Basel, Switzerland) (TABLE 2) [21].

To investigate the relationship of HER-2 overexpression and HR expression, HER-2, ER and progesterone receptors were examined as continuous variables in 1359 breast cancer specimens in 14 cell lines, some of which had been transfected with the HER-2/neu gene [22]. Transfected cell lines expressed significantly lower levels of ER and progesterone receptors than parental lines. In two clinical cohorts, primary tumors with HER-2 overexpression or pathologic amplification in the nucleus had significantly lower levels of ER and progesterone receptors than patients without HER-2 overexpression or amplification. 


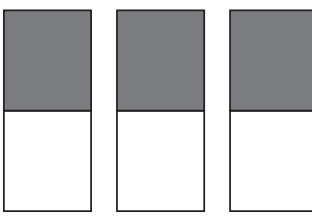

Every 3 weeks

Epirubicin $90 \mathrm{mg} / \mathrm{m}^{2}$

Cyclophosphamide $600 \mathrm{mg} / \mathrm{m}^{2}$
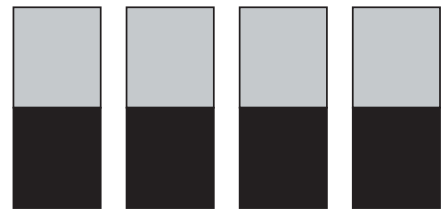

Every 3 weeks

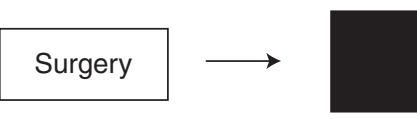

Every 3 weeks for 36 weeks

Figure 2. TECHNO trial design. Includes patients with HER2/neu 3+ or 2+ (depending on fluorescence in situ hybridization analysis result).

Therefore, pathologic HER-2 amplification and overexpression seems to downregulate expression of ER and progesterone receptors, thus impairing the efficacy of hormone therapy.

\section{Trastuzumab in the adjuvant setting}

A pilot study conducted in 40 Stage II and III breast cancer patients with preoperative weekly trastuzumab and 3-weekly paclitaxel, followed by surgery and adjuvant doxorubicin/cyclophosphamide has achieved encouraging results. Clinical response rate to preoperative therapy was $75 \%$ and complete pathologic response was $18 \%$. Therapy response was more probable for patients with HER-2 3+ tumors (84\%) than for patients with 2+ HER-2 status (38\%). Only four patients developed Grade 2 cardiotoxicity, otherwise no serious toxicity was observed [23].

Presently, several trastuzumab trials are ongoing in the adjuvant situation (BOX 1). To date, over 950 patients have been enrolled in the HERceptin Adjuvant (HERA) trial and accrual is expected to continue until 2004 and reach a total of 3192 women.

A Phase II preoperative study with epirubicin/cyclophosphamide followed by paclitaxel/trastuzumab followed by postoperative therapy with trastuzumab in patients with HER-2 overexpressing primary breast cancer has begun (TECHNO trial, by Untch $\mathrm{M}$ and coworkers) (FIGURE 2).

\section{Expert opinion}

Overexpression of the HER-2 protein is associated with aggressive tumor growth and poor prognosis. Furthermore, most patients with HER-2-overexpressing tumors benefit less from antihormonal treatment alone compared with nonoverexpressing patients. Therefore, the additional therapy option provided by trastuzumab is important for this subgroup of breast cancer patients at high risk of recurrence. Trastuzumab combines high efficacy with a toxicity profile advantageous to most patients. Except for cardiac toxicity, especially in patients who have received anthracyclines, there are almost no harmful side effects. Trastuzumab is currently approved for treatment of metastatic breast cancer and has rapidly gained a prominent role as monotherapy as well as in combination with cytostatic agents. A problem not yet solved is related to HER-2 evaluation, an essential prerequisite to any application of trastuzumab.

\section{Five-year view}

In the near future, trastuzumab may also be approved for the treatment of primary breast cancer and become a standard therapy for all breast cancer patients with HER-2-overexpressing tumors. The problem of invalid HER-2 diagnosis should be overcome, either by new and easier to apply tests or by specialized laboratories which provide complete HER-2 diagnostics. Increased knowledge of tumor biology associated with HER-2 overexpression will enable better adjustment of all therapies for high-risk breast cancers. More cancer-specific protein markers will be identified and suitable antibodies tested in clinical trials. This will bring us a big step forward in the road towards tailored breast cancer therapy.

\section{Key issues}

- Approximately 25-30\% of all breast cancer patients have tumors overexpressing the growth factor receptor HER-2.

- Overexpression of HER-2 is associated with aggressive tumors and unfavorable prognosis.

- HER-2 overexpression downregulates hormone receptor expression, thus rendering antihormonal therapy less efficient.

- Trastuzumab is a monoclonal antibody interacting with and blocking the HER-2 protein.

- Efficacy and tolerability of trastuzumab for the treatment of advanced breast cancer overexpressing HER-2 are well proven.

- Cardiac toxicity of trastuzumab limits application, especially in combination with anthracyclines. Side effects are otherwise generally mild.

- Trastuzumab has been approved for treatment of advanced breast cancer in the USA since 1998 and in Europe since 2000. Trials determining the effects of trastuzumab in primary breast cancer both before and after surgery are ongoing.

- Trastuzumab already holds a prominent place in the treatment of metastatic breast cancer overexpressing HER-2, providing a therapy option complementary to surgery, radiation, chemotherapy and antihormonal therapy. 


\section{References}

Papers of special note have been highlighted as:

- of interest

•• of considerable interest

1 Morse MA. Technology evaluation: Theratope, Biomira, Inc. Curr. Opin. Mol. Ther. 2(4), 453-458 (2000).

2 Miles DW, Towlson KE, Graham R et al. A randomised Phase II study of sialyl-Tn and detox-B adjuvant with or without cyclophosphamide pretreatment for the active specific immunotherapy of breast cancer. Br. J. Cancer 74, 1292 (1996).

3 Holmberg LA, Sandmaier BM. Theratope vaccine (STn-KLH). Exp. Opin. Biol. Ther. 1(5), 881-891 (2001).

4 Chazin VR, Kaledo M, Miller AD et al. Transformation mediated by the human HER-2 gene independent of the epidermal growth factor receptor. Oncogene 7(9), 1859-1866 (1992).

5 Pegram MD, Baly D, Wirth C. Antibody dependent cell-mediated cytotoxicity in breast cancer patients in Phase III clinical trials of humanized antiHER-2 antibody. Proc. Am. Assoc. Cancer Res. 38, 602 (1997).

6 Slamon DJ, Clark GM, Wong SG et al. Human breast cancer: correlation of relapse and survival with amplification of the HER-2/neu oncogene. Science 235, 177-182 (1987).

7 Toikkanen S, Helin H, Isola J et al. Prognostic significance of HER-2 oncoprotein expression in breast cancer: a 30-year follow-up. J. Clin. Oncol. 10, 1044-1048 (1992).

8 Buehler HJ, Kuter I, Richardson PG et al. Effective Her-2/neu diagnosis in breast cancer by a combination of immunohistochemistry and FISH. Proc. Am. Soc. Clin. Oncol. 19, 76a (2000) (Abstract 294).

9 Mass RD, Sanders C, Charlene Ket al. The concordance between the clinical trials assay (CTA) and fluorescence in situ hybridization (FISH) in the herceptin pivotal trials. Proc. Am. Soc. Clin. Oncol. 19, 75a (1999) (Abstract 291).

10 Seidman $\mathrm{AD}$, Fornire M, Esteva $\mathrm{F}$ et al. Final report: weekly herceptin and taxol for metastatic breast cancer (MBC): analysis of efficacy in Her-2/neu immunophenotype (IHC) and gene amplification (FISH). Proc. Am. Soc. Clin. Oncol. 19, 83a (2000) (Abstract 319).
11 Cobleigh A, Vogel CL, Tripathy D et al. Multinational study of the efficacy and safety of humanized antiHER-2 monoclonal antibody in women who have HER-overexpressing metastatic breast cancer that has progressed after chemotherapy for metastatic disease. $J$. Clin. Oncol. 17(9), 2639-2648 (1999).

12 Vogel CL, Cobleigh MA, Tripathy D et al. First-line herceptin monotherapy in metastatic breast cancer. Oncology 61(Suppl. 2), 37-42 (2001).

13 Vogel CL, Cobleigh MA, Tripathy D et al. Superior outcomes with herceptin (trastuzumab) in fluorescence in situ hybridization (FISH)-selected patients. Proc. Am. Soc. Clin. Oncol. 24, 86 (2001).

14 Esteva FJ, Seidman AD, Fornier M et al. Analysis of response to weekly $1 \mathrm{~h}$ taxol plus herceptin by immunophenotypic analysis in Her-2/neu overexpression (Her-2/neu +) and non-expressing (Her-2/neu-) metastatic breast cancer. Breast Cancer Res. Treat. 57, 29 (1999) (Abstract 17).

- The biggest clinical trial conducted with trastuzumab so far. It led to the US approval of trastuzumab for the treatment of advanced HER-2 overexpression breast cancer in 1998.

15 Slamon DJ, Leyland-Jones B, Shak S et al. Use of chemotherapy plus a monoclonal antibody against HER-2 for metastatic breast cancer that overexpresses HER-2. N. Engl. J. Med. 344(11) 783-792 (2001).

16 Burstein HJ, Kuter I, Campos SM et al. Clinical activity of trastuzumab and vinorelbine in women with HER-2overexpressing metastatic breast cancer. J. Clin. Oncol. 19(10), 2722-2730 (2001).

17 Konecny G, Untch M, Crohns C et al. c-erbB-2: überexpression und herceptin ${ }^{\circledR}$ in der therapie des mammakarzinoms. In: Diagnostik und Therapie des Mammakarzinoms. W Zuckschwer, München, Germany (2000).

18 Borg A, Baldetorp B, Fernö M et al. ErbB-2 amplification is associated with tamoxifen resistance in steroid-receptor positive breast cancer. Cancer Lett. 81, 137-144 (1994).

19 Ravdin PM, Green S, Albain KS et al. Initial report of the SWOG Biological Correlative Study of c-erbB-2 expression as a predictor of outcome in a trial comparing adjuvant CAF with tamoxifen (T) alone. Proc. Am. Soc. Clin. Oncol. 17, 97a (1998).
20 Mass R, Cobleigh M, Vogel C et al. Estrogen receptor status in the herceptin (trastuzumab) clinical trials: incidence and relation to clinical benefit. Proc. Am. Soc. Clin. Oncol. 24, 522 (2001).

21 Lipton A, Mouridsen H, Ali S et al. Serum Her-2/neu and response to the aromatase inhibitor letrozole versus tamoxifen. Proc. Am. Soc. Clin. Oncol. 24 (2001).

- Study assessing correlations of HER-2 overexpression and hormone receptor expression with spectacular results

22 Konecny G, Pauletti G, Pegram M et al. Quantitative association between HER$2 /$ neu and steroid hormone receptors in hormone receptor-positive primary breast cancer. J. Natl Cancer Inst. 95, 142-153 (2003).

23 Burstein HJ, Harris LN, Gelman R et al. Preoperative therapy with trastuzumab and paclitaxel followed by sequential adjuvant doxorubicin/cyclophosphamide for HER2 overexpressing Stage II or III breast cancer: a pilot study. J. Clin. Oncol. 21(1), 46-53 (2003).

\section{Affiliations}

- Michael Untch, MD Assistant Professor

Department of Gynecology and Obstetrics, Ludwig-Maximilians University, Munich, Klinikum Munich-Grosshadern, Marchioninistr. 15, D-81377,

Munich, Germany muntch@.med.uni-muenchen.de

- Nina Ditsch, $M D$ Department of Gynecology and Obstetrics, Ludwig-Maximilians University, Munich, Klinikum Munich-Grosshadern, Marchioninistr. 15, D-81377, Munich, Germany Tel.: +498970957580 Fax: +498970957582 nditsch@med.uni-muenchen.de

- Kerstin Hermelink, Dipl.-Psych Department of Gynecology and Obstetrics, Ludwig-Maximilians University, Munich, Klinikum Munich-Grosshadern, Marchioninistr. 15, D-81377, Munich, Germany Tel.: +49897095 7579 Fax: +49897095 7582 khermeli@med.uni-muenchen.de 